\title{
Model of Preformed Hole-Pairs in c-Axis Transport in Cuprate Superconductors
}

\author{
Rana Janardan Singh \\ Department of Physics, Aligarh Muslim University, Aligarh, India. \\ Email: ranajsingh@yahoo.com
}

Received August 23 ${ }^{\text {rd }}, 2012$; revised September $26^{\text {th }}, 2012$; accepted October $3^{\text {rd }}, 2012$

\begin{abstract}
Model of hole-pairs in electrical transport along ab plane in cuprate superconductors has already been proposed. It has been found to be in the shape of $3 \mathrm{~d}_{\mathrm{x} 2-\mathrm{y} 2}$ orbital of an electron in an atom. This time, model of hole-pairs in transport along c-axis in cuprate superconductors is proposed. In ab-plane, hole-pairs are formed along $\mathrm{CuO}_{2}$ plane; one hole-pair covering 9 - 10 two dimensional $\mathrm{CuO}_{2}$ unit cells in $3 \mathrm{~d}_{\mathrm{x} 2-\mathrm{y} 2}$ configuration. In the investigation of c-axis hole-pairs, cuprate superconductors have been sub-divided into three categories depending on the number of $\mathrm{CuO}_{2}$ planes/formula unit. There is a little different treatment for finding out the order parameter in each category. Coherence lengths along ab-planes are of the order of a few tens of Angstroms, whereas along c-axis, they are less than even their a-, b-lattice constants. In cuprates with 2 or $3 \mathrm{CuO}_{2}$ planes, the order parameter is of $3 \mathrm{~d}_{22-\times 2}$ type in $\mathrm{zx}$-plane with lobes along both the axes much constrained. For cuprates with a single $\mathrm{CuO}_{2}$ layer, the order parameter is of $3 \mathrm{~d}_{\mathrm{x} 2-\mathrm{y} 2}$ type, but its dimensions are less than a-, b-lattice constants.
\end{abstract}

Keywords: Cuprate Superconductors; Preformed Hole-Pairs; Electron Paramagnetic Resonance; 41 meV Peak in Neutron Diffraction

\section{Introduction}

Holes are Fermionic particles and are charge carriers in cuprate superconductors in the normal state. When two holes combine to form a Bosonic particle, they carry current in cuprate superconductors in superconducting state. Cooper pairs formed by charge carriers involving different quasi-particles have till now failed to explain high temperature cuprate superconductivity. People are veering round the idea that Bosonic pairs of charge carriers are formed much before the transition temperature and these preformed pairs undergo Bose-Einstein condensation at a lower transition temperature resulting into superconductivity.

The model of preformed hole-pairs in ab-plane of cuprate superconductors has already been given by us in the references [1,2]. The model of preformed hole-pairs in electrical conduction along c-axis of cuprate superconductors is proposed on the same lines as used for the model proposed in [2]. In [2], the positions of the two holes forming a pair at different angles $(\omega \mathrm{t}$, where $\omega=$ angular velocity of holes and $\mathrm{t}=$ time) have been expressed mathematically and results shown in a table and also shown in the Figure 1 of [2]. Ideally, this paper should be read in conjunction with [2].

However, some basic points in the derivation of $3 d_{x 2-y 2}$ order parameter in ab-plane in [2] and also the Figure 1 will be briefly presented which may be of help in understanding the present paper even in the absence of [2]. Figure 1 of [2] is reproduced here as Figure 2 to make the numbering of the rest of the figures continuous. These basic points are:

\subsection{Electron Paramagnetic Resonance Studies}

We had earlier studied electron paramagnetic resonance (EPR) of nearly all cuprate superconductors and also their constituents as $\mathrm{CuO}, \mathrm{BaCuO}_{2}, \mathrm{CaCuO}_{2}, \mathrm{SrCuO}_{2}$, $\mathrm{BiCuO}_{4}$ etc. [3-10] after deoxygenation. It may be mentioned here that cuprate superconductors as such are EPR silent because of antiferromagnetic coupling between $\mathrm{Cu}^{2+}$ ions. On deoxygenation (due to loss of oxygen from the bulk) many fragments break away and get magnetically isolated from the bulk. These fragments are $\mathrm{Cu}$ monomer, $\mathrm{Cu}$-dimer, $\mathrm{Cu}$-tetramer and $\mathrm{Cu}$-octamer. These fragments were identified on the basis of interpretation of EPR spectra. The most frequently occurring fragment was $(\mathrm{CuO})_{4}$. The spectra of $(\mathrm{CuO})_{4}$ showed that the total spin angular momentum of 4 holes associated with 4 $\mathrm{Cu}^{2+}$ ions in the $(\mathrm{CuO})_{4}$ unit $=2$, which may result from ferromagnetic coupling of the spins of $4 \mathrm{Cu}^{2+}$ ions (spin of a hole of $\mathrm{Cu}^{2+}$ ion $\left.=1 / 2\right)$. As $(\mathrm{CuO})_{4}$ is the unit cell of 
2-dimensional $\mathrm{CuO}_{2}$ sheet which is the seat of superconductivity provided a clue for understanding cuprate superconductivity. $(\mathrm{CuO})_{4}$ fragment is formed when its bonds with 8 surrounding oxygen ions in $\mathrm{CuO}_{2}$ plane are broken. $(\mathrm{CuO})_{4}$ fragment remains magnetically isolated from the bulk. As $\mathrm{Cu}-\mathrm{O}$ bond is $\approx 87.5 \%$ ionic and $\approx 12.5 \%$ covalent [11], breaking of 8 bonds amounts to removal of 1 electron from $(\mathrm{CuO})_{4}$ unit or advent of 1 hole inside it. As oxygen is more electronegative in comparison to copper, negative charge equal to that of 1 electron will be taken away by 8 oxygen ions which have broken away from the isolated $(\mathrm{CuO})_{4}$. To avoid any confusion between $\mathrm{CuO}_{2}$ and $(\mathrm{CuO})_{4}$, the difference between the two must be explained. $\mathrm{CuO}_{2}$ is the continuous sheet of copper and oxygen ions in the ab-plane of a cuprate superconductor, where each $\mathrm{Cu}^{2+}$ ion is connected to four $\mathrm{O}^{2-}$ ions. $(\mathrm{CuO})_{4}$ entity is a combination of four $\mathrm{Cu}^{2+}$ and four $\mathrm{O}^{2-}$ ions with four $\mathrm{Cu}^{2+}$ ions at the corners of a rectangular figure of dimensions equal to $a-$ and $b-$ lattice constants of the superconducting material and $\mathrm{O}^{2-}$ ions placed at the centers of four sides of the rectangle. $(\mathrm{CuO})_{4}$ remains isolated from every ion/atom in the $\mathrm{CuO}_{2}$ sheet.

\begin{tabular}{cc}
$\frac{\mathrm{HgO}}{\mathrm{HaO}}$ \\
\hline$\frac{\mathrm{BgO}}{\mathrm{BaO}}$ \\
\hline$\frac{\mathrm{CuO}}{\mathrm{CuO}}$ \\
\hline $\mathrm{BaO}$ & $\frac{\mathrm{Ca}}{\mathrm{CuO}_{2}}$ \\
\hline $\mathrm{HgO}$ & $\frac{\mathrm{Ca}}{\mathrm{CuO}_{2}}$ \\
\hline & $\frac{\mathrm{BaO}}{\mathrm{HgO}}$ \\
\hline
\end{tabular}

(a)

(b)

Figure 1. Layering schemes of (a) Hg-1201; (b) Hg-1223.

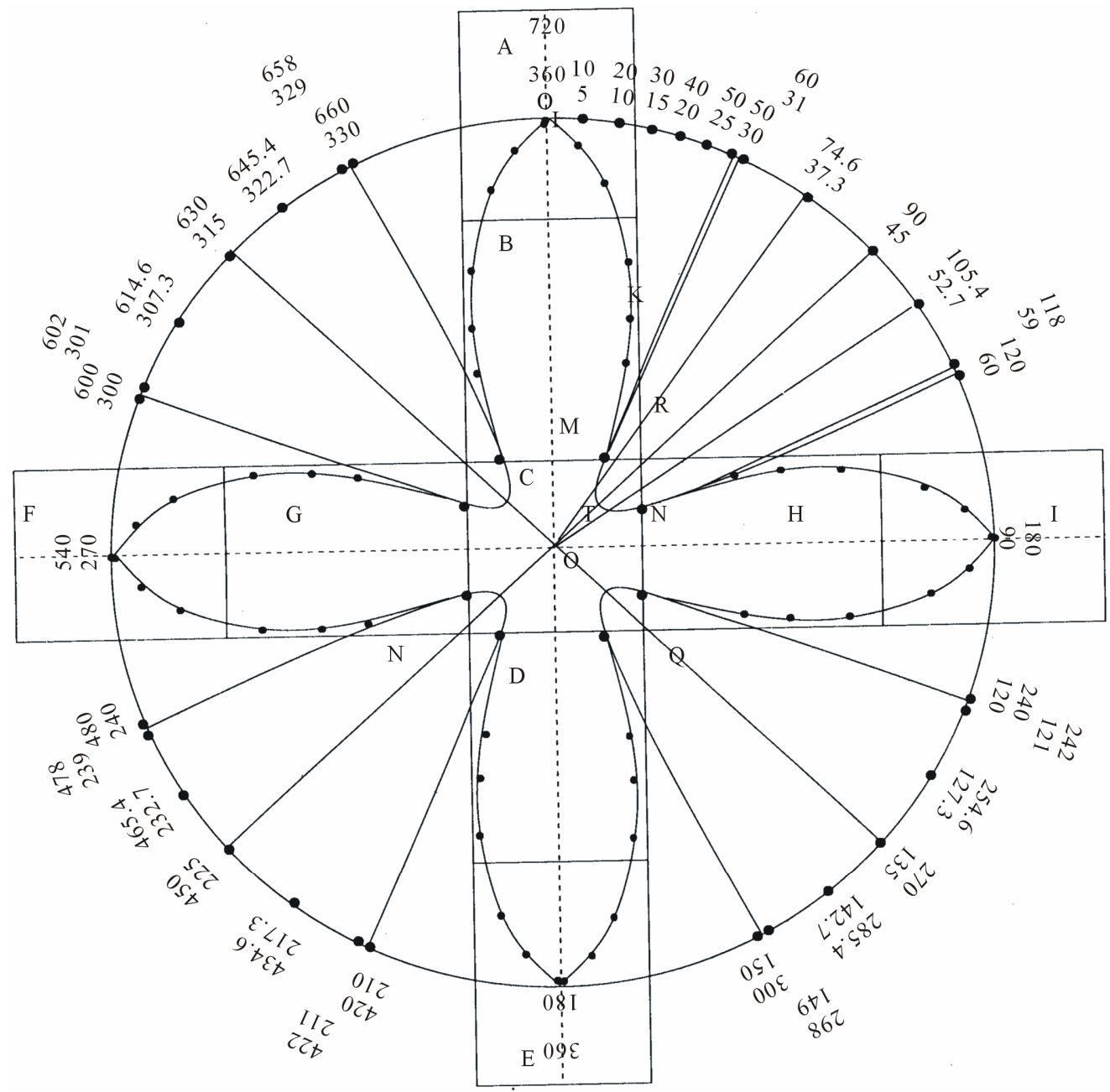

Figure 2. Model of hole-pairs in ab-plane. Both the holes traverse the same path but at any instant they are on the opposite sides of the central point. This figure is reproduction of Figure 1 from [2]. It has been reproduced to get a clearer picture of preformed hole-pairs. 
It was concluded from above arguments that even in continuous $(\mathrm{CuO})_{2}$ sheet, if a hole wandering in the $(\mathrm{CuO})_{2}$ plane on entering a $(\mathrm{CuO})_{2}$ unit cell will produce the same effect or resulting into ferromagnetic coupling of the spins of the holes of the $4 \mathrm{Cu}^{2+}$ ions. It can be said that the following equation will hold good:

An isolated $(\mathrm{CuO})_{4}$ plaquette $=\mathrm{a}(\mathrm{CuO})_{2}$ unit cell in the continuous $\mathrm{CuO}_{2}$ sheet + a hole inside it.

It has been reported in [12] that when a hole enters a $\mathrm{CuO}_{2}$ unit cell, the spins align vertically above the $\mathrm{CuO}_{2}$. The field produced will be equal to $4 \mu / \mathrm{r}^{3}$, where $\mu=$ magnetic moment of a hole and $r=$ distance from the holes. Its maximum value at the center of the plaquette $\approx$ 1900 gauss. The [12] also suggests a relation between the direction of entry of the hole in $\mathrm{a} \mathrm{CuO}_{2}$ plane and the direction of the magnetic field produced. The direction of the magnetic field produced remains at $90^{\circ}$ to the direction of entry of the hole and inclined to it in clockwise direction.

\section{2. $41 \mathrm{meV}$ Peak Observed in Neutron Diffraction and Nuclear Magnetic Resonance}

A peak at $41 \mathrm{meV}$ (with a little difference in energy from one to other superconductor) is observed in neutron diffraction and nuclear magnetic resonance spectra of high temperature superconductors. This peak is associated with spin angular momentum. $41 \mathrm{meV}$ when equated to $\mathrm{h} v, v$ comes to be equal to $10^{13}$ hertz or time-period $\mathrm{T}=$ $10^{-13} \mathrm{sec}$. We associate this frequency with oscillations of spins of $4 \mathrm{Cu}^{2+}$ holes, which means that they oscillate with this frequency from vertically upward direction to the vertically downward direction. The manner of oscillation is that when the spins are aligned vertically upward, they produce maximum magnetic field, then they distribute themselves in $\mathrm{CuO}_{2}$ plane in completely dephased manner producing zero magnetic field, and again they align in the vertically downward direction producing maximum magnetic field in the opposite direction. Again they come back to the $\mathrm{CuO}_{2}$ plane producing zero magnetic field and so on.

\subsection{Exchange Interaction}

There is always exchange interaction between two Fermions when they exist within certain separation. In the $\mathrm{CuO}_{2}$ plane when two holes come near each other from opposite sides at less than a certain distance, they are bound as a hole-pair. This pair of holes under the effect of magnetic field generated by four $\mathrm{Cu}^{2+}$ holes in the $\mathrm{CuO}_{2}$ unit cells move together in a path which is like a $3 \mathrm{~d}_{\mathrm{x} 2-\mathrm{y} 2}$ electron orbital in a atom. When the separation between the holes is very small the Coulomb repulsion between the holes will supersede exchange attraction. In $3 d_{x 2-y 2}$ path when the separation between the holes becomes large, the holes are turned back due to magnetic mirror effect. To elucidate the concept of magnetic mirror, it may be mentioned that the pole regions of the earth serve as magnetic mirrors that reflect the incoming charged particles of ionosphere and they move back and forth between two poles. The two poles of the earth act as a magnetic bottle. Mathematically also, for a charged particle moving in a magnetic field, we have $\mathrm{mv}^{2} / \mathrm{r}=\mathrm{q}$ $\mathrm{vxH}$ or $\mathrm{r}=\mathrm{mv} / \mathrm{qH}$, where $\mathrm{m}=$ mass of the particle, $\mathrm{v}=$ velocity of the particle, $q=$ charge on the particle, $r=$ radius of curvature and $\mathrm{H}=$ strength of magnetic field. The relation $\mathrm{r}=\mathrm{mv} / \mathrm{qH}$ can be written as $\mathrm{r}=\mathrm{v} / \alpha \mathrm{H}$, where $\alpha=\mathrm{q} / \mathrm{m}=$ specific charge, which is a constant quantity. In the $3 d_{x 2-y 2}$ path of the holes $v$ is constant (components $\mathrm{v}_{\mathrm{x}}$ and $\mathrm{v}_{\mathrm{y}}$ may change but not $\mathrm{v}$ by application of magnetic field). Hence from the relation, $r=v / \alpha$ $\mathrm{H}$, greater is $\mathrm{H}$, smaller is $\mathrm{r}$. At the farthest distance between the holes in the $3 d_{x 2-y 2}$ configuration the magnetic field is the highest and so the radius of curvature smallest. Therefore, the holes turn back from these positions. Then they face decreasing magnetic field and continue their journeys forward.

\subsection{Preformed Hole-Pairs Formed at Temperatures Higher than Transition Temperature}

It may be mentioned here that preformed hole pairs are formed at much higher temperatures than $T_{c}$. As the temperature is lowered their number goes on increasing. At a particular low temperature $\left(T_{c}\right)$, they undergo BoseEinstein condensation resulting into superconducting state.

For better understanding of the above ideas, the Figure 1 of [2] is reproduced below as Figure 2 in the present paper to maintain the continuity of the numbering of figures. There are many similarities and also some dissimilarities with the model we are going to give for c-axis transport.

Now we will show how the preformed hole-pairs are formed in electrical conduction along c-axis of cuprate superconductors. The shape and size of the paired charge carriers (holes) are essentially derived on the same lines as in the ab-plane as given in [2], but in details they are quite different. If one walks along the ab-plane, one finds uniform landscape consisting of $\mathrm{CuO}_{2}$ unit cells only. But if one travels along the c-axis or in a direction perpendicular to ab-plane, one has to cross through different types of terrains at nearly equal intervals. For example, if one travels along c-direction inside the Tl-2212 crystal, one has to cross through the patches consisting of $\mathrm{CuO}_{2}$, $\mathrm{Ca}, \mathrm{CuO}_{2}, \mathrm{BaO}, \mathrm{TlO}, \mathrm{TlO}, \mathrm{BaO}, \mathrm{CuO}_{2}$ unit cells as 
shown in the Figure 3(b) and repetitions of such patches. It has been reported in [13-16] that c-axis resistivity is $\approx 10^{2}$ to $10^{5}$ times larger than resistivity in ab-plane. Caxis resistivity sometimes even exceeds maximum metal resistivity of Mott-Ioffe-Regel limit. Maximum metallic resistivity is attained when mean free path of electrons $=$ interatomic distance. Crossing this limit means that mean free path is less than interatomic distance. Though c-axis resistivity $\rho_{\mathrm{c}}$ is much higher than $\rho_{\mathrm{ab}}$ in the normal state but the transition temperature $T_{c}$ remains the same in ab-plane or along c-axis. The origin of very high c-axis resistivity in normal state is that c-axis transport is metallic at higher temperatures, semiconducting at lower temperatures and again metallic before $T_{c}$ [16] but in abplane, it is metallic throughout upto $T_{c}$. In the superconducting state, the transport in ab-plane is through preformed hole-pairs and along c-axis also, transport between planes is through Josephson's tunnelling of preformed hole-pairs. These two kinds of hole-pairs along c-axis and in ab-plane are different in size and shape but are Bosonic particles and do not encounter any resistance in transportation.

Kumar et al. [13-15] gave a very reasonable explanation for highly enhanced resistivity in c-axis transport in normal state. They interpreted the suppression of single particle transport along the c-axis in the normal state as blocking of the inter-block transport by the intra-block coupling to the many body environments (i.e., entanglement with other electronic degrees of freedom). This mechanism is called Quantum Zeno Effect (QZE). However as stated earlier, the Bosonic preformed hole-pairs at $\mathrm{T}_{\mathrm{c}}$ do not suffer any scattering from the above or any other mechanism.

Now we will take up the construction, shape and size of the preformed hole-pairs in the electric transport along c-axis of cuprate superconductors that undergo BoseEinstein condensation at the transition temperature $T_{c}$, which is the main aim of this paper. Broadly, there are 5 types of high $\mathrm{T}_{\mathrm{c}}$ cuprate superconductors: 1) La-214 $\left(\mathrm{La}_{2-\mathrm{x}^{-}}\right.$ $\left.\mathrm{M}_{\mathrm{x}} \mathrm{CuO}_{4}, \mathrm{M}=\mathrm{Ba}, \mathrm{Sr}\right)$; 2) Y-123 $\left.\left(\mathrm{YBa}_{2} \mathrm{Cu}_{3} \mathrm{O}_{7-\delta}\right) ; 3\right)\left(\mathrm{Tl}_{2}\right.$ $\mathrm{Ba}_{2} \mathrm{Ca}_{\mathrm{n}-1} \mathrm{Cu}_{\mathrm{n}} \mathrm{O}_{2 \mathrm{n}+4}$ with $\left.\mathrm{n}=1,2,3\right)$; 4) $\left(\mathrm{Bi}_{2} \mathrm{Sr}_{2} \mathrm{Ca}_{\mathrm{n}-1} \mathrm{Cu}_{\mathrm{n}} \mathrm{O}_{\mathrm{x}}\right.$ with $\mathrm{n}=1,2,3)$; and 5) $\left(\mathrm{HgBa}_{2} \mathrm{Ca}_{\mathrm{n}-1} \mathrm{Cu}_{\mathrm{n}} \mathrm{O}_{\mathrm{y}}\right.$ with $\mathrm{n}=1$ and 3 ).

The properties of the last three superconductors are more anisotropic than the first two. $\mathrm{CuO}_{2}$ is common among all of them. The layering schemes of all the superconductors have been given in the Figures 1 and 3-5. The main properties of superconductors have been given in the Table 1.

It is well known that in superconductors, $\mathrm{CuO}_{2}$ layers

\begin{tabular}{c}
\hline $\mathrm{CuO}_{2}$ \\
\hline $\mathrm{BaO}$ \\
\hline $\mathrm{TlO}$ \\
\hline $\mathrm{TlO}$ \\
\hline $\mathrm{BaO}$ \\
\hline $\mathrm{CuO}_{2}$ \\
\hline $\mathrm{BaO}$ \\
\hline $\mathrm{TlO}$ \\
\hline $\mathrm{TlO}$ \\
\hline $\mathrm{BaO}^{\mathrm{CuO}_{2}}$ \\
\hline
\end{tabular}

(a)

\begin{tabular}{c}
\hline $\mathrm{Ca}$ \\
\hline $\mathrm{CuO}_{2}$ \\
\hline $\mathrm{BaO}$ \\
\hline $\mathrm{TlO}$ \\
\hline $\mathrm{TlO}$ \\
\hline $\mathrm{BaO}$ \\
\hline $\mathrm{CuO}_{2}$ \\
\hline $\mathrm{Ca}$ \\
\hline $\mathrm{CuO}$ \\
\hline $\mathrm{BaO}$ \\
\hline $\mathrm{TlO}$ \\
\hline $\mathrm{TlO}$ \\
\hline $\mathrm{BaO}$ \\
\hline $\mathrm{CuO}$ \\
\hline $\mathrm{Ca}$ \\
\hline
\end{tabular}

(b)

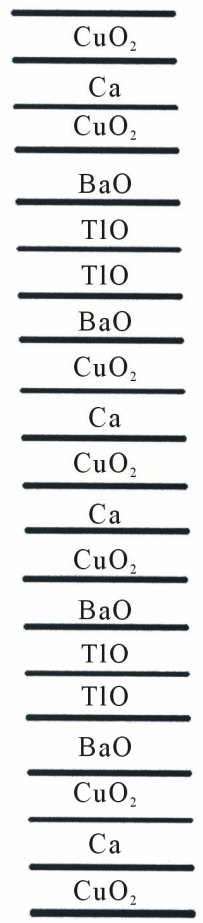

(c)

Figure 3. Layering schemes of (a) Tl- 2201, (b) Tl-2212, (c) Tl-2223. Note: Layering schemes of Bi-2201, Bi-2212 and Bi-2223 are similar to those of their Tl-counterparts with the difference that instead of $\mathrm{Tl}$ and $\mathrm{Ba}$ in $\mathrm{Tl}$-superconductors, Bi and $\mathrm{Sr}$ are substituted in Bi-superconductors. Another important difference occurring in Bi-superconductors is that in the packing of layers along c-axis, two layers of $\mathrm{BiO}$ separated by $3 \AA$ are shifted relative to each other along the diagonal direction of the perovskite sub-cell. 


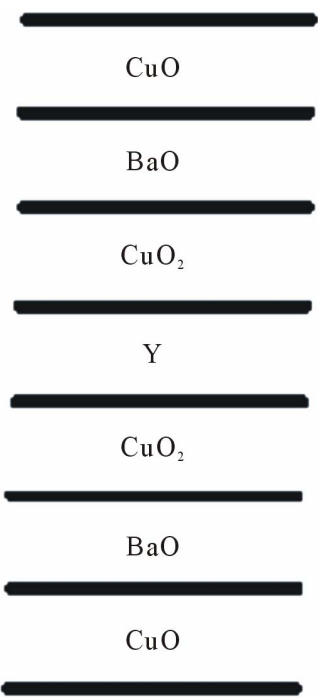

Figure 4. Layering scheme of Y-123.

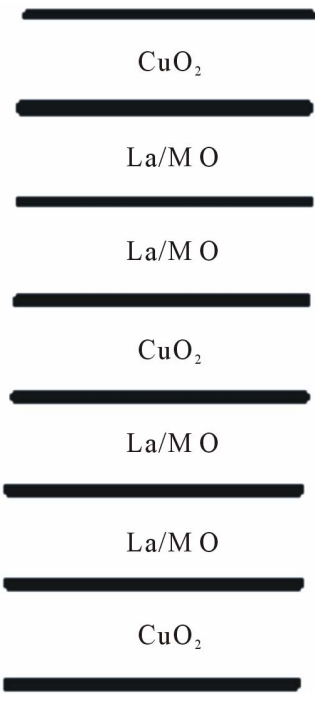

Figure 5. Layering scheme of La-214.

Table 1. Some important data about superconductors. ${ }^{36} \mathrm{Kr}_{84}=1 s^{2} 2 s^{2} 2 p^{6} 3 s^{2} 3 p^{6} 3 d^{10} 4 s^{2} 4 p^{6}{ }^{54}{ }^{5} e_{131}=1 s^{2} 2 s^{2} 2 p^{6} 3 s^{2} 3 p^{6} 3 d^{10} 4 s^{2} 4 p^{6}$ $4 d^{10} 5 s^{2} 5 p^{6}$. O denotes partial filling.

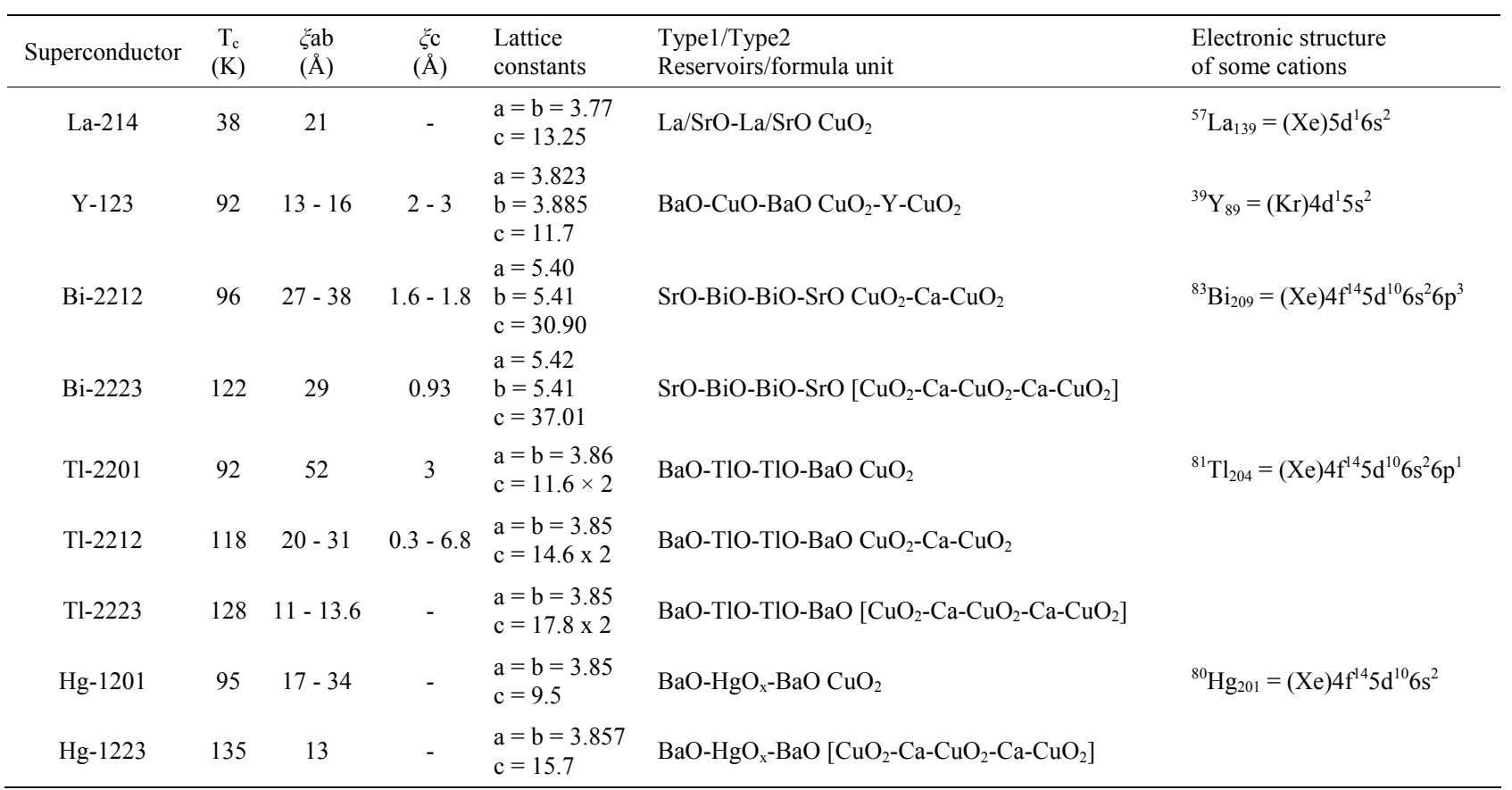

are the seat of superconductivity and they govern most of the properties. So depending upon the number of $\mathrm{CuO}_{2}$ layers in the unit cell of a superconductor, they have been divided into three categories: 1) Having 3 layers of $\mathrm{CuO}_{2}$ as in $\mathrm{Tl}_{2} \mathrm{Ba}_{2} \mathrm{Ca}_{2} \mathrm{Cu}_{3} \mathrm{O}_{\mathrm{x}}, \mathrm{Bi}_{2} \mathrm{Sr}_{2} \mathrm{Ca}_{2} \mathrm{Cu}_{3} \mathrm{O}_{\mathrm{y}}$, $\mathrm{HgBa}_{2} \mathrm{Ca}_{2} \mathrm{Cu}_{3} \mathrm{O}_{z} ; 2$ ) 2 layers of $\mathrm{CuO}_{2}$ as in $\mathrm{Tl}_{2} \mathrm{Ba}_{2} \mathrm{CaCu}_{2} \mathrm{O}_{\mathrm{x}}$, $\mathrm{Bi}_{2} \mathrm{Sr}_{2} \mathrm{CaCu}_{2} \mathrm{O}_{\mathrm{y}}, \mathrm{YBa}_{2} \mathrm{Cu}_{3} \mathrm{O}_{7-\delta}$; 3) 1 layer of $\mathrm{CuO}_{2}$ as in $\mathrm{Tl}_{2} \mathrm{Ba}_{2} \mathrm{CuO}_{\mathrm{x}}, \mathrm{HgBa}_{2} \mathrm{CuO}_{\mathrm{z}}, \mathrm{La}_{1-\mathrm{x}} \mathrm{M}_{\mathrm{x}} \mathrm{CuO}_{4}$ (where $\mathrm{M}=\mathrm{Ba}$ or $\mathrm{Sr})$.

Before going further with the discussion of hole-pair formation, we will establish clear distinction between two kinds of holes which have been mentioned earlier in the text and will often come in our discussion to avoid any confusion. One kind of hole is that which is generated in the superconductors due to the level of doping and which is the electric current carrier in superconductors and it will be called lattice hole. The other kind of holes is that which is associated with $\mathrm{Cu}^{2+}$ ions in $\mathrm{CuO}_{2}$ network and which generates magnetic field on arrival of a lattice hole in the $\mathrm{CuO}_{2}$ unit cell. This kind of holes will be called $\mathrm{Cu}^{2+}$ holes.

First we discuss the representative case of $\mathrm{Tl}_{2} \mathrm{Ba}_{2} \mathrm{Ca}$ - 
$\mathrm{Cu}_{2} \mathrm{O}_{\mathrm{x}}$, in the category (2) consisting of 2 layers of $\mathrm{CuO}_{2}$. In all our discussions, we will be mainly interested in giving the shape, size and symmetry of the preformed hole-pairs (i.e., order parameter) in transport along c-axis and estimating the value of $\xi_{\mathrm{c}}$ (coherence length along c-axis). In Tl-2212, there are two $\mathrm{CuO}_{2}$ layers or blocks and in between them there is one layer containing $\mathrm{Ca}$ as shown in the Figure 3(b) [Layer is the common term but it is generally used for 2-dimensional objects. Along the $\mathrm{c}$-axis, there are 3-dimensional blocks of $\mathrm{TlO}, \mathrm{BaO}$, $\mathrm{CuO}_{2}$ etc. heaped one over the other. So in our discussion, both the terms can be used as synonyms]. $\mathrm{As}^{\mathrm{CuO}_{2}}$ layers are most active layers, therefore, the hole pair is expected to be formed in between these two layers or in the block containing Ca.

We now explain the formation of hole-pairs shown in the Figure 6(a). The coordinate system for the Figure 6 has been given in the Figure 6(c).

There are two lattice holes, one approaching from above and the other from below. Only the motion of the hole 1 starting from position A in the figure will be described. Same kind of description is true for the hole 2 starting from $\mathrm{E}$ in the figure. The hole 2 is always situated diagrammatically opposite to the hole 1 because of starting from the opposite side. The motion of the two holes is guided by the three effects mentioned in the beginning, 1) magnetic field generated by the advent of a hole inside a $\mathrm{CuO}_{2}$ block; 2) oscillations of spins of holes of four $\mathrm{Cu}^{2+}$ ions with a frequency of $10^{13}$ hertz which produce varying magnetic field with the same frequency. Figure 6(b) shows the variation of magnetic field from a maximum to zero, then from zero to the maximum but in opposite direction and then again to zero as shown in Parts A, B, C, D respectively; 3) Both the holes are always under the effect of exchange interaction which varies with separation between the holes.

It needs to be mentioned here that the motion of a charged hole in a magnetic field is governed by right hand rule. Hole 1 starting from A travels with its thermal velocity towards the Ca-block but not in straight line but along the curved path $\mathrm{AB}$ (Figure 6(a)) due to the magnetic field direction shown in the Figure 6(b)(A). Then it is deflected along the path $\mathrm{BC}$ due to Coulomb repulsion from $\mathrm{Ca}^{2+}$ ion and also from the hole 2 which has simultaneously reached near the $\mathrm{Ca}^{2+}$ ion at $\mathrm{F}$ on the opposite side of hole1. At this instant the magnetic field seems to have nearly vanished (Figure 6(b)(B)) and the Coulomb repulsion seems to have superseded attractive exchange interaction. Charge distribution on the $\mathrm{Ca}^{2+}$ ion seems to act as reflecting planes for holes 1 and 2 when they reached the positions at $\mathrm{B}$ and $\mathrm{F}$ respectively. From B, the hole 1 reaches $\mathrm{C}$ under the effect of increasing magnetic field in the downward direction. From $\mathrm{C}$ the hole 1 reverses its direction of motion and follows the path $\mathrm{C}$ to

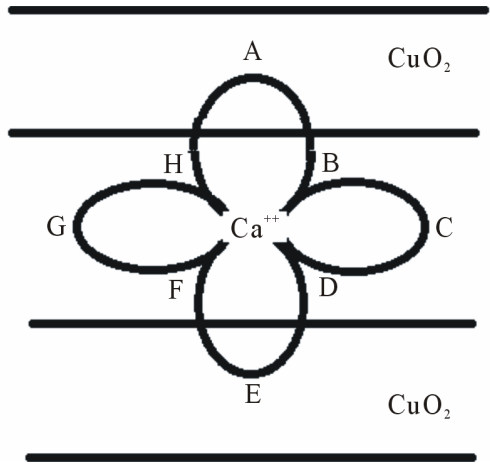

(a)
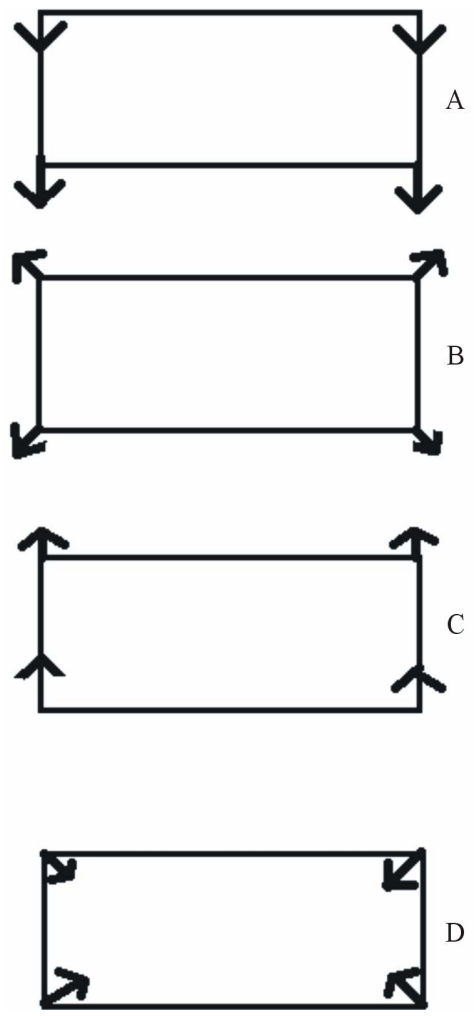

(b)

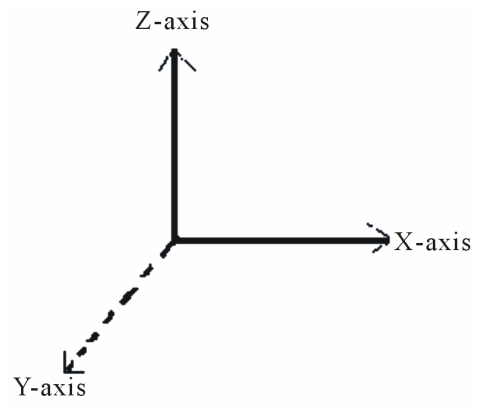

(c)

Figure 6. (a) Model of hole-pairs in c-axis transport; (b) Directions of magnetic field produced by spin alignments of $4 \mathrm{Cu}^{2+}$ ions of $\mathrm{CuO}_{2}$ unit cell. From $\mathrm{A}$ to $\mathrm{B}$ to $\mathrm{C}$ to $\mathrm{D}$ to $\mathrm{A}$ again, the spin arrangements have been shown at intervals of $T / 4$. Time-period of spin oscillation $(T)=10^{-13} \mathrm{sec}$; (c) Coordinate system for all figures under Figure 6. 
D. At $\mathrm{C}$ the magnetic field is maximum but vertically downward and at $\mathrm{D}$, the magnetic field becomes nearly zero. The important question is how the path of the hole 1 is reversed? The reason is the magnetic mirror effect. Magnetic mirror effect has been explained earlier. At D, again the Coulomb repulsion supersedes exchange interaction and charge distribution on $\mathrm{Ca}^{2+}$ acts like a reflecting plane and directs hole 1 towards E. From D, the hole 1 reaches $\mathrm{E}$ under the effect of magnetic field increasing vertically upwards. In the time the hole 1 has reached $\mathrm{E}$ through the path $\mathrm{ABCDE}$, the hole 2 has reached $\mathrm{A}$ through the path EFGHA. The hole 2 experiences same kind of forces as the hole 1. Both the holes are always situated diametrically opposite to each other in the path ABCDEFGHA. The two holes continue moving along the path $A B C D E F G H A$ and form a hole-pair.

In the journey of hole 1 from $A$, there are 4 stations at $\mathrm{B}, \mathrm{C}, \mathrm{D}$ and at $\mathrm{E}$. At each station, there is drastic change in the path of the hole 1 . The question is what is special about these stations? As have been mentioned above, these are the positions from where magnetic field changes from maximum at $\mathrm{A}$ to minimum or zero at $\mathrm{B}$, then maximum at $\mathrm{C}$ but in the opposite direction, then zero at $\mathrm{D}$ and again maximum at $\mathrm{E}$ in the same direction as at A. In the journey of lattice hole from A to E, the spins of $\mathrm{Cu}^{2+}$ holes have completed one full oscillation. It means that time taken by the hole 1 is $10^{-13} \mathrm{sec}$. as the frequency of oscillations of spins of $\mathrm{Cu}^{2+}$ holes which cause magnetic field $=10^{13}$ hertz. Therefore, the time for the hole 1 to complete each segment of its journey A to B; $\mathrm{B}$ to $\mathrm{C}$; $\mathrm{C}$ to $\mathrm{D}$ and $\mathrm{D}$ to $\mathrm{E}$ should be the same and equals to $1 / 4 \times 10^{-13} \mathrm{sec}$. For completing each segment of its journey, it travels with its thermal velocity and for equal time of $1 / 4 \times 10^{-13} \mathrm{sec}$. Hence all the segments should also be of the same length. The same is true for the hole 2. Thus it can be said that in coming from A to E, spins have completed one oscillation or tilted by $360^{\circ}$, though the change of geometrical angle $=180^{\circ}$ only as seen from the Figure 6(a) and also Figure 2.

The shape of the hole-pair shown in the Figure 6(a) is $3 \mathrm{~d}_{\mathrm{z} 2-\mathrm{x} 2}$ because the coordinates involved are $\mathrm{z}$-axis and $\mathrm{x}$-axis. It is not of usual $3 \mathrm{~d}_{\mathrm{x} 2-\mathrm{y} 2}$ type obtained for the hole pair in ab-plane of superconductors. The dimensions of $3 d_{x 2-y 2}$ hole pairs in ab plane is usually a few tens of Angstroms. But along c-axis, the dimensions are usually less than a- and b-lattice constants. The reason for the small dimension of hole-pairs along c-axis transport may be explained as follows. We have seen that the length of every limb of every $3 d_{z 2-x 2}$ hole pair is decided by the distance travelled by a hole in time $=1 / 4 \times 10^{-13} \mathrm{sec}$. or equals to velocity of the hole $\mathrm{x} 1 / 4 \times 10^{-13} \mathrm{sec}$. Because of incoherent in-plane scattering [13-15], the velocity of hole in the $\mathrm{x}$-direction is much reduced. Also the velocity of the hole in the z-direction or along the c-axis is also much reduced due to uneven terrain and also due to the Quantum Zeno Effect (QZE) [13-15] which suppresses c-axis transport due to intra-block incoherent scattering. It may be possible that limbs of hole-pairs in z- and $\mathrm{x}$-directions may be of different length depending upon the resistivity along the $\mathrm{z}$ - and $\mathrm{x}$-directions. It is well known that greater is the resistivity of a material, less is the velocity of charge carriers. Because the velocities of holes along x- or z-direction are not known exactly, it can not be said which limb is bigger and which shorter. But it is known that both limbs will be constrained in comparison to $3 \mathrm{~d}_{\mathrm{x} 2-\mathrm{y} 2}$ hole-pairs in ab-plane.

Now we discuss $\mathrm{Tl}_{2} \mathrm{Ba}_{2} \mathrm{Ca}_{2} \mathrm{Cu}_{3} \mathrm{O}_{\mathrm{x}}$ (others in the class being $\mathrm{Bi}_{2} \mathrm{Sr}_{2} \mathrm{Ca}_{2} \mathrm{Cu}_{3} \mathrm{O}_{\mathrm{y}}$ and $\mathrm{HgBa}_{2} \mathrm{Ca}_{2} \mathrm{Cu}_{3} \mathrm{O}_{\mathrm{z}}$ ) which has three bocks of $\mathrm{CuO}_{2}$ and two intervening blocks of $\mathrm{Ca}$ as shown in the Figure 3(c). In this case there are two pairs of $\mathrm{CuO}_{2}$ layers each containing a $\mathrm{Ca}$ layer in between. One pair consists of upper and middle $\mathrm{CuO}_{2}$ layer with a $\mathrm{Ca}$ layer in between, the other pair consists of the middle and the lower $\mathrm{CuO}_{2}$ layer with another $\mathrm{Ca}$ layer in between. Two holes coming from opposite sides, one appearing in the upper $\mathrm{CuO}_{2}$ layer and the other appearing in the middle layer simultaneously will form a hole-pair in the intervening Ca block in the same manner as explained in the case of Tl-2212 discussed above. In the other pair of $\mathrm{CuO}_{2}$ layers consisting of the middle and the lower $\mathrm{CuO}_{2}$ blocks with a $\mathrm{Ca}$ block in between, a hole-pair will be formed in the same way as in the first pair. Definitely, there is more probability of hole-pairs in Tl-2223 than in Tl-2212, which should ensure higher $T_{c}$ for Tl-2223 than for Tl-2212, which is corroborated by experiment. $\mathrm{T}_{\mathrm{c}}$ of Tl-2223 $=128 \mathrm{~K}$ and $\mathrm{T}_{\mathrm{c}}$ for Tl-2212 $=$ $118 \mathrm{~K}$.

Next we discuss $\mathrm{Tl}_{2} \mathrm{Ba}_{2} \mathrm{CuO}_{\mathrm{x}}$ (or Tl-2201). Others in this class are $\mathrm{HgBa}_{2} \mathrm{CuO}_{\mathrm{z}}$ and $\mathrm{La}_{2-\mathrm{x}} \mathrm{M}_{\mathrm{x}} \mathrm{CuO}_{4}$, where $\mathrm{M}=$ $\mathrm{Ba}$ or $\mathrm{Sr}$. In this system, there is only one $\mathrm{CuO}_{2}$ layer bounded on both sides by four blocks of $\mathrm{BaO}, \mathrm{TlO}, \mathrm{TlO}$, $\mathrm{BaO}$ as shown in the Figure 3(a). If two holes, one from above and one from below reach the $\mathrm{CuO}_{2}$ block simultaneously, both will induce the spins of four $\mathrm{Cu}^{2+}$ holes in the $\mathrm{CuO}_{2}$ unit to align in the A-configuration [12] of the Figure 6(b). and then oscillate from one configuration to the other (i.e., $\mathrm{A}$ to $\mathrm{B}$ to $\mathrm{C}$ to $\mathrm{D}$ to $\mathrm{A}$ ). In the same way as in Tl-2212, the two holes under the action of the magnetic field produced from the fluctuating spins of the four $\mathrm{Cu}^{2+}$ ions and exchange effects will follow a closed path in the plane of the $\mathrm{CuO}_{2}$ layer, i.e., in the ab-plane or xy-plane. The symmetry of the order parameter will be of $3 \mathrm{~d}_{\mathrm{x} 2-\mathrm{y} 2}$ and not $3 \mathrm{~d}_{\mathrm{z2}-\mathrm{x} 2}$ as in Tl-2212. The hole-pairs along c-axis transport in other cases discussed here have been found to be of $3 \mathrm{~d}_{\mathrm{z2}-\mathrm{x} 2}$ type but in the case of Tl-2201 and its associates, it is of type $3 \mathrm{~d}_{\mathrm{x} 2-\mathrm{y} 2}$. It has been found experimentally that transport in Tl-2201 is metallic both in ab-plane and along c-axis. There arises an obvi- 
ous question. If in Tl-2201, the order-parameter both in ab-plane and along c-axis is of $3 \mathrm{~d}_{\mathrm{x} 2-\mathrm{y} 2}$ type and transport in both case being metallic, why there should be so much difference between $\xi_{\mathrm{ab}}(=52 \AA)$ and $\xi_{\mathrm{c}}(=3 \AA)$. It should be because of different paths taken in current flow in ab-plane and along c-axis. Conduction in the ab-plane is smooth consisting of homogeneous $\mathrm{CuO}_{2}$ unit cells, but conduction along c-axis is through heterogeneous slabs of $\mathrm{BaO}, \mathrm{TlO}, \mathrm{TlO}, \mathrm{BaO}$ and their repetitions. Moreover, Quantum Zeno Effect (QZE) discussed earlier will be effective in reducing the velocity of hole in x-direction due to incoherent scattering.

From the Table 1, it is noticed that $\xi_{\mathrm{ab}}$ are of the order of tens of Angstroms, but $\xi_{\mathrm{c}}$ is less that a- and b-lattice constants except in Tl-2212, where it has been reported to vary from 0.3 to $6.8 \AA$. Such large variation of $\xi_{\mathrm{c}}$ seems to be due to some kind of experimental error. In [13-18], it has been pointed out that it is quite possible for any measurement of resistivity $\rho_{\mathrm{c}}$ to pick up some in-plane component of resistive tensor-perhaps externally due to misalignment of contacts or internally due to the randomly distributed defects.

There are two more points worth considering: 1) Why $\mathrm{T}_{\mathrm{c}}$ of a superconductor increases when number of $\mathrm{CuO}_{2}$ layers per unit cell increases (see Table 1) from one to three. It is because more the number of $\mathrm{CuO}_{2}$ layers, greater will be the number of hole-pairs formed and transition temperature will be reached at higher temperature. Legget [19] has shown that for identically doped members of the same homologous series, as a result of the inter-plane Coulomb interaction, the difference $\Delta \mathrm{T}_{\mathrm{c}}{ }^{(\mathrm{n})}$ of the transition temperature for the nth member from the single layer value is given by $\Delta \mathrm{T}_{\mathrm{c}}^{(\mathrm{n})}=$ Constant $(1-1 / \mathrm{n})$. This value of $\Delta \mathrm{T}_{\mathrm{c}}^{(\mathrm{n})}$ is to be added to the single layer $\mathrm{T}_{\mathrm{c}}$.

Ratan Lal et al., [20] on the basis of phenomenological arguments and structural features have inferred that $\mathrm{Cu}$ $3 \mathrm{~d}_{322-\mathrm{r} 2}$ orbitals are involved in the transport along c-axis. This kind of orbital may be correct for single particle transport in normal state. But the question with which we are concerned here is to find out what is the symmetry of the hole-pair in the electrical transport along c-axis at $T_{c}$ and below. It has been found out here that symmetry of the hole-pair carrying current along $\mathrm{c}$-axis at $\mathrm{T}_{\mathrm{c}}$ or below is of $3 \mathrm{~d}_{22-\times 2}$ type for $\mathrm{n}$ (number of $\mathrm{CuO}_{2}$ layers/unit cell) $=2$ and 3 and of $3 d_{x 2-y 2}$ type for $\mathrm{n}=1$.

\section{Summary}

The model of preformed hole-pairs in ab-plane of cuprate superconductors has been proposed earlier, which is of symmetry $3 \mathrm{~d}_{\mathrm{x} 2-\mathrm{y} 2}$. The model of hole-pairs in the electrical transport along c-axis at $T_{c}$ and below has been proposed in this paper. The models for c-axis transport have been proposed in three sections corresponding to the num- ber of layers $n=1,2,3$ per formula unit. For $n=2$ and 3 , the symmetry of the preformed pairs is of $3 \mathrm{~d}_{\mathrm{z2}-\mathrm{x} 2}$ type (i.e., in $\mathrm{zx}$-plane). But for $\mathrm{n}=1$, the symmetry of the order parameter is of $3 \mathrm{~d}_{\mathrm{x} 2-\mathrm{y} 2}$ type. The reasons leading to very small coherence length in c-axis transport have been given. It is suggested to carry out angle-dependent experimental studies on cuprate superconductor single crystals as thermal conductivity, specific heat, STM imaging etc to verify the proposed hole-pair models.

\section{REFERENCES}

[1] R. J. Singh, "Preformed Hole Pairs in Cuprate Superconductors," International Journal of Modern Physics B, Vol. 23, No. 1, 2009, pp. 53-76.

[2] R. J. Singh, "Model of Preformed Hole-Pairs in Cuprate Superconductors," Journal of Modern Physics, Vol. 2, No. 8, 2011, pp. 53-76.

[3] A. Punnoose, B. P. Maurya, J. Mathew, M. Umar, M. I. Haque and R. J. Singh, "EPR Observation of $\mathrm{Cu}^{2+}-\mathrm{Cu}^{2+}$ Pairs in Cupric Oxide Powders," Solid State Communications, Vol. 88, No. 3, 1993, pp. 195-198.

[4] R. J. Singh, A. Punnoose, J. Mathew, B. P. Maurya, et al., " $S=1$ and $S=2$ EPR Signals in Modified $\mathrm{CuO}$ and $\mathrm{BaCuO}_{2}$," Physical Review B, Vol. 49, No. 2, 1994, pp. 1346-1349. doi:10.1103/PhysRevB.49.1346

[5] R. J. Singh, M. Ikram, A. Punnoose, B. P. Maurya and S. Khan, "Copper Tetramers in High-Temperature Superconductors," Physics Letters A, Vol. 208, No. 4, 1995, pp. 369-374.

[6] A. Punnoose and R. J. Singh, "EPR Studies of High- $\mathrm{T}_{\mathrm{c}} \mathrm{Su}-$ perconductors and Related Systems," International Journal of Modern Physics, Vol. 9, No. 10, 1995, pp. 1123-1157. doi:10.1142/S0217979295000471

[7] S. Khan, M. Ikram, A. Singh and R. J. Singh, "EPR Study of Deoxygenated $\mathrm{La}_{2} \mathrm{CuO}_{4}$," Physica C, Vol. 281, No. 2-3, 1997, pp. 143-148. doi:10.1016/S0921-4534(97)00328-6

[8] S. Khan, A. Singh and R. J. Singh, "EPR Study of $\mathrm{La}_{2-\mathrm{x}} \mathrm{Sr}_{\mathrm{x}} \mathrm{CuO}_{4}[\mathrm{M}=\mathrm{Ba}, \mathrm{Sr}]$," Solid State Communications, Vol. 106, No. 9, 1998, pp. 621-626.

[9] S. Khan, A. Singh and R. J. Singh, "EPR Study of $\mathrm{La}_{1.854} \mathrm{Sr}_{0.146} \mathrm{CuO}_{4}$," Physica C, Vol. 325, 1999, pp. 165-172. doi:10.1016/S0921-4534(99)00513-4

[10] R. J. Singh, P. K. Sharma, A. Singh and S. Khan, "EPR Spectra of Deoxygenated High Temperature Superconductors," Physica C, Vol. 356, No. 4, 2001, pp. 285-296. doi:10.1016/S0921-4534(01)00283-0

[11] Q. B. Meng, Z. J. Wu and S. Y. Zhang, "Evaluation of the Energy Barrier Distribution in Many-Particle Systems Using the Path Integral Approach," Journal of Physics: Condensed Matter, Vol. 10, No. 5, 1998, pp. L85-L88.

[12] P. C. Dai, H. A. Mook, G. Aeppli, S. M. Hayden and F. Dogan, "Resonance as a Measure of Pairing Correlations in the High- $\mathrm{T}_{\mathrm{c}}$ Superconductor $\mathrm{YBa}_{2} \mathrm{Cu}_{3} \mathrm{O}_{6.6}$," Nature, Vol. 406, No. 6799, 2000, pp. 965-968. doi: $10.1038 / 35023094$ 
[13] N. Kumar, T. P. Pareek and A. M. Jayannavar, "Normal State c-Axis Resistivity of the High- $\mathrm{T}_{\mathrm{c}}$ Cuprate Superconductors," 1997.

[14] N. Kumar, "The Zeno Effect and Interlayer Pairing Mechanism for High Temperature Superconductivity in Layered Materials," 2000.

[15] N. Kumar and A. M. Jayannavar, "Temperature Dependence of the c-Axis Resistivity of High- $\mathrm{T}_{\mathrm{c}}$ Layered Oxides," Physical Review B, Vol. 45, No. 9, 1992, pp. 50015004.

[16] K. Takenaka, M. Mizuhasi, H. Takagi and S. Uchida, "Interplane Charge Transport in $\mathrm{YBa}_{2} \mathrm{Cu}_{3} \mathrm{O}_{7-\mathrm{y}}$," Physical Review B, Vol. 50, No. 9, 1994, pp. 6534-6537.

[17] Y. C. Ma, J. W. Liu, H. W. Lu and H. L. Zheng, "Out of Plane Temperature Dependent Resistivity Studies on Tl-
Based Superconductors," Journal of Physics: Condensed Matter, Vol. 19, No. 18, 2007, pp. 186203-186209. doi:10.1088/0953-8984/19/18/186203

[18] R. Jin, D. P. Grandatto and H. R. Rott, "Normal State Resistivity of Superconducting $\mathrm{Bi}_{1.95} \mathrm{Sr}_{1.65} \mathrm{La}_{0.4} \mathrm{CuO}_{6+\delta}$," Physica C, Vol. 250, No. 3, 1995, pp. 395-402. doi:10.1016/0921-4534(95)00369-X

[19] A. J. Legget, "Cuprate Superconductivity: Dependence of $\mathrm{T}_{\mathrm{c}}$ on the c-Axis Layering Structure," Physical Review Letters, Vol. 83, No. 2, 1999, pp. 392-395. doi:10.1103/PhysRevLett.83.392

[20] R. Lal, Ajay, R. L. Hotta and S. K. Joshi, "Model for c-Axis Resistivity in Cuprate Superconductors," Physical Review B, Vol. 75, No. 10, 1998, pp. 6126-6136. 